\title{
ON SECOND-HARMONIC GENERATION IN NONPARABOLIC QUANTUM WELLS
}

\author{
M. ZALUŻNY \\ Institute of Physics, M. Curie-Skłodowska University \\ Pl. M. Curie-Skłodowskiej 1, 20-031 Lublin, Poland
}

The second-harmonic generation due to the intersubband transitions in nonparabolic two-level quantum well systems is discussed theoretically taking into account the depolarization effect.

PACS numbers: 78.66.Fd, 42.65.Ky

The second-order nonlinear optical properties connected with the intersubband transitions in asymmetric quantum wells (AQWs) have been investigated experimentally and theoretically by several groups [1-3]. In most of the papers the authors concentrate on the systems with parabolic subbands and neglect the dynamical screening. The effect of this screening on the linear response is well known. In systems with parallel subbands it leads to a depolarization shift between the intersubband spacing and the intersubband infrared absorption resonance $[4,5]$. However, what is less known is that the depolarization effect (DE) modifies also the absorption line shape when the subband separation depends on the wave vector due to the nonparabolicity of the constituent materials. Calculations reported in Refs. [6-8] indicate that the contribution to the line broadening resulting from the nonparabolicity is, to a large extent, compensated by the DE.

The second-harmonic generation (SHG) spectrum is also affected by the DE $[3,9,10]$. It has been shown experimentally [3] that in AQWs with two parallel subbands the resonance in $\chi^{(2)}(2 \omega)$ occurs when $2 \hbar \omega$ (or $\hbar \omega$ ) coincides with the depolarization shifted intersubband energy, and not the bar intersubband spacing.

In this note we present preliminary calculations indicating that, like in the case of the linear response, the DE leads to a line narrowing of the SHG spectrum for the two-subband nonparabolic system. (The case of the doubly-resonant three-level system will be discussed in a separate paper.)

Our analysis is based on the density matrix formulation in the electric dipole approximation similar to that used in our previous papers $[10,11]$.

In a reference system with the $z$-axis normal to the surface the eigenfunction of the effective mass Hamiltonian describing the electron in the $j$-th subband can be written as $\exp \left(\mathrm{i} k_{\|} r_{\|}\right) \Psi_{j, k_{\|}}(z)$ where $k_{\|}$and $r_{\|}$are the wave vector and the position vector in the $x-y$ plane. When the nonparabolicity is not 
very strong the multidimensional vector of envelope function $\Psi_{j, k_{\|}}(z)$ can be replaced, in the first approximation [12], by the (normalized) conduction component $\varphi_{j}(z) \equiv \varphi_{j, k_{\|}=0}(z)$ which is the solution of the one-dimensional Schrödinger equation $\left[p_{z}^{2} / 2 m+V(z)\right] \varphi_{j}(z)=E_{j} \varphi_{j}(z) . E_{j}$ is the minimum energy of the $j$-th subband and $m$ is the energy- and position-dependent effective mass $[6,12]$. The nonparabolicity of the constituent materials leads to the nonparabolicity in the energy dispersion relation $E_{j}(k l e l)$ for electrons in the $j$-th subband. Consequently, the energy separation between the subbands $E_{21}\left(k_{\|}\right)\left[=E_{2}\left(k_{\|}\right)-E_{1}\left(k_{\|}\right)\right]$depends (1) $k_{\|}$. (For the semiquantitative estimation the above dependence can be calculated along the line of our implementation [6] of the empirical two-band model proposed in Ref. [13].)

The equation of motion for the matrix elements of the density operator $\rho$ [in the representation of $\left.\left|j, k_{\|}\right\rangle=\exp \left(i k_{\|} r_{\|}\right) \varphi_{j}(z)(j=1,2)\right]$ is given by

$$
\frac{\partial \rho_{i j}}{\partial t}=\frac{1}{\mathrm{i} \hbar}\left[H_{0}+V, \rho\right]_{i j}-\frac{\Delta \rho_{i j}}{\tau_{i j}}
$$

where $H_{0}$ is the one-band unperturbed Hamiltonian, $V \equiv V(z, t)$ is the effective perturbing Hamiltonian, $\tau_{i i}^{-1}$ is the relaxation rate from the $i$-th subband, $\tau_{i j}^{-1}$ is the off-diagonal elastic dephasing rate and $\Delta \rho=\rho-\rho^{(0)}$, where $\rho^{(0)}$ is the unperturbed density matrix. The diagonal element $\rho_{j j}^{(0)} \equiv \rho_{j k_{\| j} k_{\|}}^{(0)}$ equals to the thermal equilibrium occupation probability $\left[F_{j}\left(k_{\|}\right)\right]$of the corresponding state. The equilibrium surface density of the electrons in the $j$-th subband is given by $N_{j}=2 \sum_{k_{\|}} F_{j}\left(k_{\|}\right)$. (In this paper the explicit reference to the dependence of the matrix elements of $\rho$ on $k_{\|}$is omitted for brevity.)

The electric field $E(t)=E(\omega) \exp (-\mathrm{i} \omega t)+$ c.c. of the pumped radiation (applied in the $z$-direction) modifies the density distribution of electrons. This leads to the modification of the effective perturbing potential $V(z, t)=V^{\operatorname{ext}}(z, t)+$ $V^{\text {ind }}(z, t)$ where $V^{\text {ext }}(z, t)=e E(t) z$ is the external perturbation. $V^{\text {ind }}(z, t)(=$ the potential induced in the system) is given (in the electrostatic limit) by the solution of Poisson's equation with the change of the distribution $\Delta n(z, t)$ as the source term $[5,10]$.

As in most of the previous papers we assume that $V^{\text {ext }}(z, t)$ is sufficiently small. Then, the surface electronic polarization of the AQW can be expanded in powers of $E$. If we shall limit ourselves to the first two orders and neglect the rectification effect [3] then

$$
P_{s}(t)=\epsilon_{0} \chi^{(1)}(\omega) E(\omega) \exp (-\mathrm{i} \omega t)+\epsilon_{0} \chi^{(2)}(2 \omega) E(\omega)^{2} \exp (-\mathrm{i} 2 \omega t)+\text { c.c. },
$$

where $\chi^{(1)}(\omega)$ and $\chi^{(2)}(2 \omega)$ are the linear and SHG coefficients (per unit surface), respectively.

Employing the similar expansion for $\Delta \rho, V$ and $\Delta n$ we get (for details see Refs. $[10,11])$ the following expression for the $n$-th-order surface electronic susceptibility

$$
\chi^{(n)}\left(\omega_{n}\right)=\frac{-e}{\epsilon_{0} E^{n}(\omega)} 2 \sum_{k_{\|}} \sum_{i, j} \rho_{i j}^{(n)}\left(\omega_{n}\right) z_{j i},
$$


where $z_{i j}=z_{j i}=\int_{-\infty}^{\infty} \mathrm{d} z \varphi_{i}(z) z \varphi_{j}(z)$ and $\omega_{n}=n \omega \cdot \rho_{i j}^{(n)}\left(\omega_{n}\right)$ is defined by equation

$$
\begin{gathered}
\rho_{i j}^{(n)}\left(\omega_{n}\right)=\frac{1}{\hbar \omega_{n}-E_{i j}\left(k_{\|}\right)+\mathrm{i} \Gamma_{i j}}\left\{\sum_{k=1}^{n-1}\left[V^{(k)}\left(z, \omega_{k}\right), \rho^{(n-k)}\left(\omega_{n-k}\right)\right]_{i j}\right. \\
\left.+\frac{e^{2}}{\epsilon_{0} \epsilon_{\infty}} \sum_{m, p} L(i, j ; m, p) \bar{\rho}_{m p}^{(2)}\left(\omega_{n}\right) F_{i j}\left(k_{\|}\right)\right\},
\end{gathered}
$$

with

$$
V_{i j}^{(n)}\left(\omega_{n}\right)=V_{i j}^{\mathrm{ext}}(\omega) \delta_{1 n}+\frac{e^{2}}{\epsilon_{0} \epsilon_{\infty}} \sum_{k, l} L(i, j ; k, l) \bar{\rho}_{k l}^{(n)}\left(\omega_{n}\right),
$$

where $L(i, j ; k, l)$ is the Coulomb matrix element defined in Ref. [4], $\Gamma_{i j}=\hbar \tau_{i j}^{-1}$, $\bar{\rho}_{i j}^{(n)}\left(\omega_{n}\right)=2 \sum_{k_{\|}} \rho_{i j}^{(n)}\left(\omega_{n}\right)$ and $F_{i j}\left(k_{\|}\right)=F_{i}\left(k_{\|}\right)-F_{j}\left(k_{\|}\right)$.

The expression for $\chi^{(1)}(\omega)$ resulting from Eqs. (3)-(5) takes the form

$$
\chi^{(1)}(\omega)=\frac{e^{2}\left(z_{21}\right)^{2} N_{12}}{\epsilon_{0}} \frac{2}{E_{21}} G(\omega)
$$

where $G(\omega)=L(\omega) /[1+\alpha L(\omega)]$ with

$$
\begin{aligned}
& L(\omega)=\frac{1}{N_{12}} \sum_{k_{\|}} \frac{2 F_{12}\left(k_{\|}\right) E_{21} E_{21}\left(k_{\|}\right)}{E_{21}\left(k_{\|}\right)^{2}-(\hbar \omega+\mathrm{i} \Gamma)^{2}} \\
& \approx \frac{1}{N_{12}} \sum_{k_{\|}} \frac{F_{12}\left(k_{\|}\right) 2 E_{21}^{2}}{E_{21}\left(k_{\|}\right)^{2}-(\hbar \omega+\mathrm{i} \Gamma)^{2}} .
\end{aligned}
$$

Here

$$
\begin{aligned}
& \alpha=\frac{2 e^{2} N_{12}}{\epsilon_{0} \epsilon_{\infty} E_{21}} L(1,2 ; 1,2)=\frac{2 e^{2} N_{12}}{\epsilon_{0} \epsilon_{\infty} E_{21}} \int_{-\infty}^{\infty} \mathrm{d} z\left[\int_{-\infty}^{z} d z^{\prime} \varphi_{1}\left(z^{\prime}\right) \varphi_{2}\left(z^{\prime}\right)\right]^{2}, \\
& N_{i j}=N_{i}-N_{j}, \quad \Gamma=\Gamma_{12}=\Gamma_{21}
\end{aligned}
$$

and

$$
E_{21}=E_{21}(0) \text {. }
$$

One can check that if we neglect the nonparabolicity of the subbands $\left[E_{21}\left(k_{\|}\right)=E_{21}\right]$ then $G(\omega)=E_{21}^{2} /\left[\tilde{E}_{21}^{2}-(\hbar \omega+\mathrm{i} \Gamma)^{2}\right]$ where $\tilde{E}_{21}=E_{21}(1+\alpha)^{1 / 2}$ is the depolarization shifted intersubband energy $[4,5]$.

We find from Eq. (6) that in the presence (absence) of the DE the linear absorption spectrum is determined by $\operatorname{Im} G(\omega)[\operatorname{Im} L(\omega)]$ (see Refs. [6-8]).

To obtain a simple analytical expression for $\chi^{(2)}(2 \omega)$ we have to made several approximations. Following [1] and [10] we restrict to the near resonant situation $2 \hbar \omega \approx E_{21}$ and assume that $\alpha \ll 1$, i.e. $E_{21} \approx \tilde{E}_{21}$. In this limit one can neglect the contribution of the nonresonant terms $\rho_{i i}^{(2)}(2 \omega)$ and $\rho_{12}^{(2)}(2 \omega)$ to $\chi^{(2)}(2 \omega)$ [see Eq. (3)]. Moreover, calculating the nonresonant terms $V_{i j}^{(1)}(\omega)$ and $\rho_{i j}^{(1)}(\omega)$ one can 
neglect the $\mathrm{DE}$ and replace $E_{21}\left(k_{\|}\right)$by $E_{21}$. Employing the above simplifications we get the following expression for the $\mathrm{SH}$ susceptibility:

$$
\chi^{(2)}(2 \omega)=\frac{-e^{3} N_{12}}{\epsilon_{0}} \frac{\left(z_{22}-z_{11}\right) z_{21}^{2}}{E_{21}-\hbar \omega-\mathrm{i} \Gamma} \frac{2}{E_{21}} G^{\prime}(2 \omega),
$$

where $G^{\prime}(2 \omega)=L^{\prime}(2 \omega) /\left[1+\alpha L^{\prime}(2 \omega)\right]$ with

$$
L^{\prime}(2 \omega)=\frac{1}{N_{12}} \sum_{\boldsymbol{k}_{\|}} \frac{F_{12}\left(k_{\|}\right) E_{21}}{E_{21}\left(k_{\|}\right)-2 \hbar \omega-\mathrm{i} \Gamma} .
$$

In the case of the parabolic subbands $G^{\prime}(2 \omega)=E_{21} /\left[2\left(E_{21}-2 \hbar \omega-\mathrm{i} \Gamma\right)\right]$ where $\bar{E}_{21}=E_{21}(1+\alpha / 2)$. Note that in the approximation used here $(\alpha \ll 1)$ the difference between $\tilde{E}_{21}$ and $\bar{E}_{21}$ is negligible. Consequently, the difference between $G(2 \omega)$ and $G^{\prime}(2 \omega)$ is also small (when $\hbar \omega$ is close to $E_{21} / 2$ ).

The SIIG spectrum is proportional to $\left|\chi^{(2)}(2 \omega)\right|^{2}$ [1]. Thus, in contrast with the linear case, the line shape is now determined mainly by $\left|G^{\prime}(2 \omega)\right|^{2}$, not by $\operatorname{Im} G^{\prime}(2 \omega)[\approx \operatorname{Im} G(2 \omega)]$. However, our calculations show that the difference in the spectral shape between $\operatorname{Im} G^{\prime}(2 \omega)$ and $\left|G^{\prime}(2 \omega)\right|^{2}$ is not substantial. This means that the SII generation spectrum is affected by the DE in the similar way as the linear absorption spectrum i.e., the peak value (line width) of $\left|G^{\prime}(2 \omega)\right|^{2}$ is larger (smaller) than the peak value (line width) of $\left|L^{\prime}(2 \omega)\right|^{2}$. As an illustration we present (see Fig. 1) the $\omega$ dependence of $\left|\bar{G}^{\prime}(2 \omega)\right|^{2}=\left|G^{\prime}(2 \omega)\right|^{2}\left(2 \Gamma / E_{21}\right)^{2}$

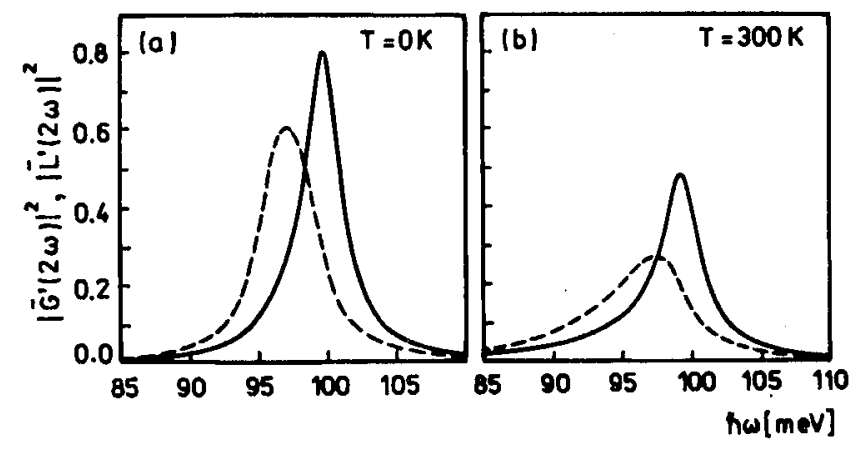

Fig. 1. The spectral dependence of $\left|\bar{G}^{\prime}(2 \omega)\right|^{2}$ (solid lines) and $\left|\bar{L}^{\prime}(2 \omega)\right|^{2}$ (dashed lines) for the step GaAs AQW described in the text: (a) $T=0 \mathrm{~K}$ and (b) $T=300 \mathrm{~K}$.

and $\left|\bar{L}^{\prime}(2 \omega)\right|^{2}=\left|\bar{L}^{\prime}(2 \omega)\right|^{2}\left(2 \Gamma / E_{21}\right)^{2}$ calculated at $T=0 \mathrm{~K}$ and $T=300 \mathrm{~K}$ for $\mathrm{Al}_{0.4} \mathrm{Ga}_{0.6} \mathrm{As} / \mathrm{GaAs} / \mathrm{Al}_{0.28} \mathrm{Ga}_{0.72} \mathrm{As} / \mathrm{Al}_{0.4} \mathrm{Ga}_{0.6} \mathrm{As} \mathrm{AQW}$ structure with the $\mathrm{GaAs}$ well thickness of $35 \AA$, the total well thickness of $70 \AA, \Gamma=3 \mathrm{meV}, N_{1}=$ $10^{12} \mathrm{~cm}^{-2}$ and $N_{2}=0$. (The above structure is nearly optimized [1] for response at $\hbar \omega \approx 100 \mathrm{meV}$.) For the $\mathrm{GaAs} / \mathrm{Al}_{x} \mathrm{Ga}_{1-x} \mathrm{As}$ parameters we used: the interface energy barrier $\Delta E_{\mathrm{c}}(x)=0.7 \Delta E_{\mathrm{g}}(x)$ where $\Delta E_{\mathrm{g}}(x)[\mathrm{eV}]=1.425 x-0.9 x^{2}+$ $1.1 x^{3} ; m_{\mid \mathrm{Al}_{x} \mathrm{Ga}_{1-x} \mathrm{As}}=\left[0.0665+0.0835 \Delta E_{\mathrm{g}}(x) / 1.625\right] m_{0}$ and $\gamma=4.9 \times 10^{-19} \mathrm{~m}^{2}$ for the nonparabolicity coefficient [13]. (Following [6] and [7] we neglect the temperature dependence of the above parameters.) 
From the obtained results we can conclude that in the case of two-subband nonparabolic systems the DE leads not only to the shift of the peak in the SHG spectrum but also induces the line narrowing and the enhancement of the peak value. Preliminary calculations indicate that similar narrowing and enhancement should also take place in the double resonant three-subband systems.

\section{References}

[1] E. Rosencher, Ph. Bois, Phys. Rev. B 44, 11315 (1991) and references therein.

[2] F. Capasso, C. Sirtori, Y. Cho, IEEE J. Quantum Electron. 30, 1313 (1994).

[3] J.N. Heyman, K. Craig, B. Galdrikian, M.S. Sherwin, K. Campman, P.F. Hopkins, S. Fafard, A.C. Gossard, Phys. Rev. Lett. 72, 2183 (1994).

[4] S.J. Allen, D.C. Tsui, B. Vinter, Solid State Commun. 20, 425 (1976).

[5] T. Ando, A. Fowler, F. Stern, Rev. Mod. Phys. 54, 437 (1982).

[6] M. Załużny, Phys. Rev. B 43, 4511 (1991).

[7] M. Załużny, Phys. Rev. B 49, 2923 (1994).

[8] R.J. Warburton, C. Gauer, A. Wixforth, J.P. Kotthaus, B. Brar, H. Kroemer, Superlattices Microstruct., in press.

[9] A. Liu, O. Keller, Proc. SPIE 2139, 342 (1994).

[10] M. Zalużny, Phys. Rev. B 51, 9757 (1995).

[11] M. Zalużny, V. Bondarenko, J. Appl. Phys. 79, 6750 (1996).

[12] C. Sirtori, F. Capasso, J. Faist, S. Scandalo, Phys. Rev. B 50, 8663 (1994).

[13] D.F. Nelson, R.C. Miller, D.A. Kleimann, Phys. Rev. B 35, 7770 (1987). 